\title{
Outgrowth of a CSF3R-mutant clone drives a second myeloproliferative neoplasm in a chronic myeloid leukemia patient: a case report
}

\author{
Sarah A. Carratt ${ }^{1,2}$, Diana Brewer ${ }^{1,2}$, Julia E. Maxson ${ }^{1,2}$, Brian J. Druker ${ }^{1,2}$ and Theodore P. Braun ${ }^{1,2^{*}}$
}

\begin{abstract}
Background: Chronic myeloid leukemia (CML) and chronic neutrophilic leukemia (CNL) are two myeloproliferative neoplasms with mutually exclusive diagnostic criteria. A hallmark of CML is the Philadelphia chromosome (Ph), which results in a BCR-ABL1 fusion gene and constitutive tyrosine kinase activity. CNL is a Ph-negative neoplasm and is defined in part by the presence of CSF3R mutations, which drive constative JAK/STAT signaling.

Case presentation: Here, we report the exceedingly rare co-occurrence of two granulocytic myeloproliferative neoplasms in a 69-year old male patient. After an initial diagnosis of chronic myeloid leukemia, the patient's clinical course was shaped by hematologic toxicity, the emergence of treatment-resistant BCR-ABL1 clones, and the expansion of a CSF3R-mutant clone without ABL1 mutations under selective pressure from tyrosine kinase inhibitors. The emergence of the CSF3R-mutant, neutrophilic clone led to the diagnosis of CNL as a second myeloproliferative neoplasm in the same patient.

Conclusions: This is the first reported case of CNL arising subsequent to CML, which occurred under selective pressure from targeted therapy in a patient with complex clonal architecture. Patients with such molecularly complex disease may ultimately benefit from combination therapy that targets multiple oncogenic pathways.
\end{abstract}

Keywords: Case report, Chronic myeloid leukemia, Chronic neutrophilic leukemia, Myeloproliferative neoplasm, Tyrosine kinase inhibitors, Clonal evolution

\section{Background}

Myeloproliferative neoplasms (MPNs) are clonal hematologic malignancies in which a hematopoietic stem cell defect drives proliferation of mature myeloid cell lineages. Chronic myeloid leukemia (CML) and chronic neutrophilic leukemia (CNL) are two MPNs with mutually exclusive diagnostic criteria. A hallmark of CML is the presence of the Philadelphia $(\mathrm{Ph})$ chromosome, which

\footnotetext{
* Correspondence: braunt@ohsu.edu

'Knight Cancer Institute, Oregon Health \& Science University, Portland, OR 97239, USA

${ }^{2}$ Division of Hematology and Medical Oncology, Oregon Health \& Science University, Portland, OR 97239, USA
}

results from $t(9 ; 22)$ and separates $C M L$ from Ph-negative MPNs: essential thrombocythemia, primary myelofibrosis, polycythemia vera, chronic neutrophilic leukemia [1]. The BCR-ABL1 fusion gene results from $t(9 ; 22)$ and drives the proliferation of mature myeloid cells through constitutive tyrosine kinase activity [2, 3]. In this report, we describe a second rare MPN, chronic neutrophilic leukemia $(\mathrm{CNL})$, arising after a CML patient achieved a complete molecular remission. This is the first reported case of CNL arising subsequent to CML.

(c) The Author(s). 2021 Open Access This article is licensed under a Creative Commons Attribution 4.0 International License, which permits use, sharing, adaptation, distribution and reproduction in any medium or format, as long as you give appropriate credit to the original author(s) and the source, provide a link to the Creative Commons licence, and indicate if changes were made. The images or other third party material in this article are included in the article's Creative Commons licence, unless indicated otherwise in a credit line to the material. If material is not included in the article's Creative Commons licence and your intended use is not permitted by statutory regulation or exceeds the permitted use, you will need to obtain permission directly from the copyright holder. To view a copy of this licence, visit http://creativecommons.org/licenses/by/4.0/ The Creative Commons Public Domain Dedication waiver (http://creativecommons.org/publicdomain/zero/1.0/) applies to the data made available in this article, unless otherwise stated in a credit line to the data. 


\section{Case presentation}

A 69-year-old presented initially to his primary care provider with a progressive cough accompanied by weakness, fatigue, and abdominal distension. His white cell count was $113,900 / \mathrm{mm}^{3}$, hemoglobin was $11.8 \mathrm{~g} / \mathrm{dL}$, and platelet count was $325,000 / \mathrm{mm}^{3}$. A bone marrow biopsy showed a markedly hypercellular marrow (100\%) with $4 \%$ blasts, consistent with chronic phase CML. A 300 cell differential showed left-shifted myelopoiesis in which mid-range differentiated granulocytes-myelocytes, metamyelocytes with lesser amounts of promyelocytes-predominated over more differentiated forms. Myeloid and erythroid precursors were present in a 20:1 ratio. Auer rods were not observed. A Fluorescent In Situ Hybridization (FISH) assay confirmed a BCR-ABL1 translocation and cytogenetics revealed the Ph chromosome resulting from a $(9 ; 22)$ translocation. The major BCR-ABL1 transcript (p210) was $66.45 \%$ on the international scale (IS). The patient was started on $300 \mathrm{mg}$ of nilotinib twice daily, which reduced his BCR-ABL1 to $0.403 \%$ (IS) in 18 weeks. However, the emergence of six new pathogenic mutations as well as hematologic toxicity of ABL1 inhibitors in this case made the treatment course complex (Tables 1,2).

After 18 weeks on nilotinib, the patient presented with chest pain and was found to have pericarditis, atrial fibrillation, and severe thrombocytopenia (platelets at 11,000). The patient was ultimately started on imatinib at $400 \mathrm{mg} /$ day but thrombocytopenia remained problematic. After 2 months, imatinib was reduced to $300 \mathrm{mg} /$ day in an attempt to stabilize the patient's ongoing thrombocytopenia. His platelets only slightly improved, however BCR-ABL1 transcripts decreased. The $400 \mathrm{mg} /$ day dose was resumed when BCR-ABL1 rose from 0.68 to $2.74 \%$ (IS); however, subsequent $\mathrm{ABL}$ sequencing detected an imatinib and nilotinib-resistant E255V mutation [4], and the patient switched to dasatinib at $100 \mathrm{mg} /$ day.

The leukemia responded well to dasatinib, though BCR-ABL1 transcript level plateaued above $0.1 \%$ (IS), the threshold for a major molecular response. ABL sequencing after 9 months of treatment detected a T315I mutation, which confers resistance to dasatinib, nilotinib, imatinib and bosutinib [5-11]. The patient was switched to ponatinib (30 mg/day) and his BCR-ABL1 transcript levels decreased steadily, falling below $0.1 \%$ (IS) after 3 months. The patient tolerated ponatinib well, with no thrombocytopenia. After 2 years on ponatinib, the $(9 ; 22)$ translocation was not detected by FISH in 200 cells scored for BCR (22Q11.2)/ABL (9Q34). Additionally, the BCR-ABL1 fusion gene was no longer detectable by PCR, $0.000 \%$ (IS). After 2.5 years, the dose of ponatinib was reduced to $15 \mathrm{mg} /$ day.

Despite the disappearance of the $\mathrm{Ph}$ chromosome, the patient's white blood cell and absolute neutrophil counts rose while on ponatinib, raising concerns about whether a second Ph-negative MPN could be emerging (Fig. 1). A 220 gene next generation sequencing panel was ordered and two CSF3R mutations, T618I and W818*, were identified at a variant allele frequency (VAF) of 41.1 and $40.3 \%$, respectively. Predicted pathogenic variants were identified based on the COSMIC v92 (Catalogue of Somatic Mutations in Cancer) database. Retrospective analysis of an archived marrow sample revealed that the CSF3R mutations had been present for at least the past 3 years. Bone marrow from diagnosis was not available for retrospective next generation sequencing analyses. In total, six predicted-pathogenic variants were detected in the patient over the course of his treatment, including two CSF3R mutations (T618I and W818*), two ABL1 mutations (E255V and T315I), KMT2C/MLL3 (S1860C), and TET2 (R1167K) (Table 2).

CSF3R mutations are a hallmark of chronic neutrophilic leukemia (CNL), a rare MPN defined by persistent mature neutrophilic leukocytosis. When the CSF3R variants were detected, the patient's WBC was $22,800 / \mathrm{mm}^{3}$ with $83 \%$ neutrophils, within the range of WHO-defined CNL $\left(11,000-126,000 / \mathrm{mm}^{3}\right.$, median $\left.39,000 / \mathrm{mm}^{3}\right)$. The patient was started on ruxolitinib (5 $\mathrm{mg}$ twice daily), which demonstrated efficacy against CNL and CSF3Rmutant atypical CML in a recent clinical trial $[12,13]$. With ruxolitinib, the patient's WBC count decreased slightly and stabilized between $12,800-17,400 / \mathrm{mm}^{3}$, absolute neutrophils between 9700 and $14,300 / \mathrm{mm}^{3}$, platelets were stable at $124,000-155,000 / \mathrm{mm}^{3}$, hemoglobin at

Table 1 Treatment strategy. The patient had difficulty tolerating TKls due to hematologic toxicity (thrombocytopenia). Additionally, identification of three genetic variants triggered three treatment changes: imatinib and nilotinib-resistant BCR-ABL1 E255V, ponatinib-sensitive BCR-ABL1 T315I, ruxolitinib-sensitive CSF3R mutations. Dates initiated and discontinued are the number of weeks post-diagnosis of CML

\begin{tabular}{lllll}
\hline Inhibitor & Molecular Target & Initiated & Discontinued & Reason Discontinued \\
\hline Nilotinib & BCR-ABL1 & $0 \mathrm{w}$ & $28 \mathrm{w}$ & Thrombocytopenia \\
Imatinib & BCR-ABL1 & $33 \mathrm{w}$ & $78 \mathrm{w}$ & BCR-ABL1 p.E255V \\
Dasatinib & BCR-ABL1 & $79 \mathrm{w}$ & $118 \mathrm{w}$ BCR-ABL1 p.T315। \\
Ponatinib & BCR-ABL1 & $128 \mathrm{w}$ & \\
Ruxolitinib & CSF3R & $252 \mathrm{w}$ & \\
\hline
\end{tabular}


Table 2 Variant allele frequencies and BCR-ABL transcript levels 3 years apart for predicted pathogenic mutations. Outgrowth of a CSF3R-mutant clone during treatment with BCR-ABL1 tyrosine kinase inhibitors drives disease evolution from CML to CNL

\begin{tabular}{|c|c|c|c|c|c|}
\hline \multirow[t]{2}{*}{ Gene } & \multirow[t]{2}{*}{ Variant } & \multirow{2}{*}{$\begin{array}{l}\text { COSMIC v92 } \\
\text { FATHMM prediction }\end{array}$} & \multicolumn{3}{|c|}{ Variant Allele Frequency } \\
\hline & & & 69 weeks & 116 weeks & 225 weeks \\
\hline KMT2C/MLL3 & S1860C & Pathogenic (score 0.90) & 50.62 & & 48.92 \\
\hline CSF3R & T618। & Pathogenic (score 0.96) & 11.58 & & 41.09 \\
\hline CSF3R & W818* & Pathogenic (score 0.83) & 10.22 & & 40.26 \\
\hline TET2 & R1167K & Pathogenic (score 0.99) & 2.86 & & \\
\hline ABL1 & E255V & (not annotated) & 2.01 & Not detected (targeted PCR) & \\
\hline ABL1 & T3151 & (not annotated) & & Detected (targeted PCR) & \\
\hline BCR-ABL1 & & & $2.50 \%(I S)$ & $0.508 \%(I S)$ & $0.000 \%$ (IS) \\
\hline
\end{tabular}

15.3-16.6 g/dL, hematocrit at 46.6-51\%. Ruxolitinib was increased to $7.5 \mathrm{mg}$ twice daily after 7 months, which lowered his WBC (8900-13,000/ $\left.\mathrm{mm}^{3}\right)$ and absolute neutrophil $\left(6300-10,000 / \mathrm{mm}^{3}\right)$ counts further. At 5.8 years post CML diagnosis and 3.5 years on ponatinib, a third FISH analysis was performed and the $(9 ; 22)$ translocation was still not detectable (200 nuclei).

\section{Discussion and conclusions}

This patient had difficulty tolerating tyrosine kinase inhibitors (TKIs), primarily due to hematologic toxicity. While thrombocytopenia is a known side effect of TKIs, the severity and protracted nature of the side effect in this instance suggests dysfunction in the residual BCRABL1 negative stem cells. In this case, the emergence of six predicted-pathogenetic variants over the five-year course of treatment suggests that the patient either had a markedly elevated mutational load prior to diagnosiswith different clones emerging in response to TKI selection-or a propensity to develop mutations beyond what is normally associated with aging.

The mutations in BCR-ABL1 and TET2 were present at similar variant allele frequencies at 69 weeks and lost during treatment with dasatinib and ponatinib, suggesting they were present in the same clone. Meanwhile, the CSF3R-mutant clone expanded during BCR-ABL1-directed therapy and is likely independent. Given that the increase in neutrophils began when the patient was switched from dasatinib to ponatinib, it is plausible that the expansion of this clone occurred during treatment with ponatinib specifically. It is known that CSF3R truncating mutations are sensitive to dasatinib [14], and that may have contributed to a delayed onset of this second MPN.

It is well established that selective pressure from TKIs allows for the outgrowth of BCR-ABL1 clones harboring point mutations in ABL1. However, this study suggests that the clearance of a BCR-ABL1 positive clone allowed for the outgrowth of a second, genetically distinct

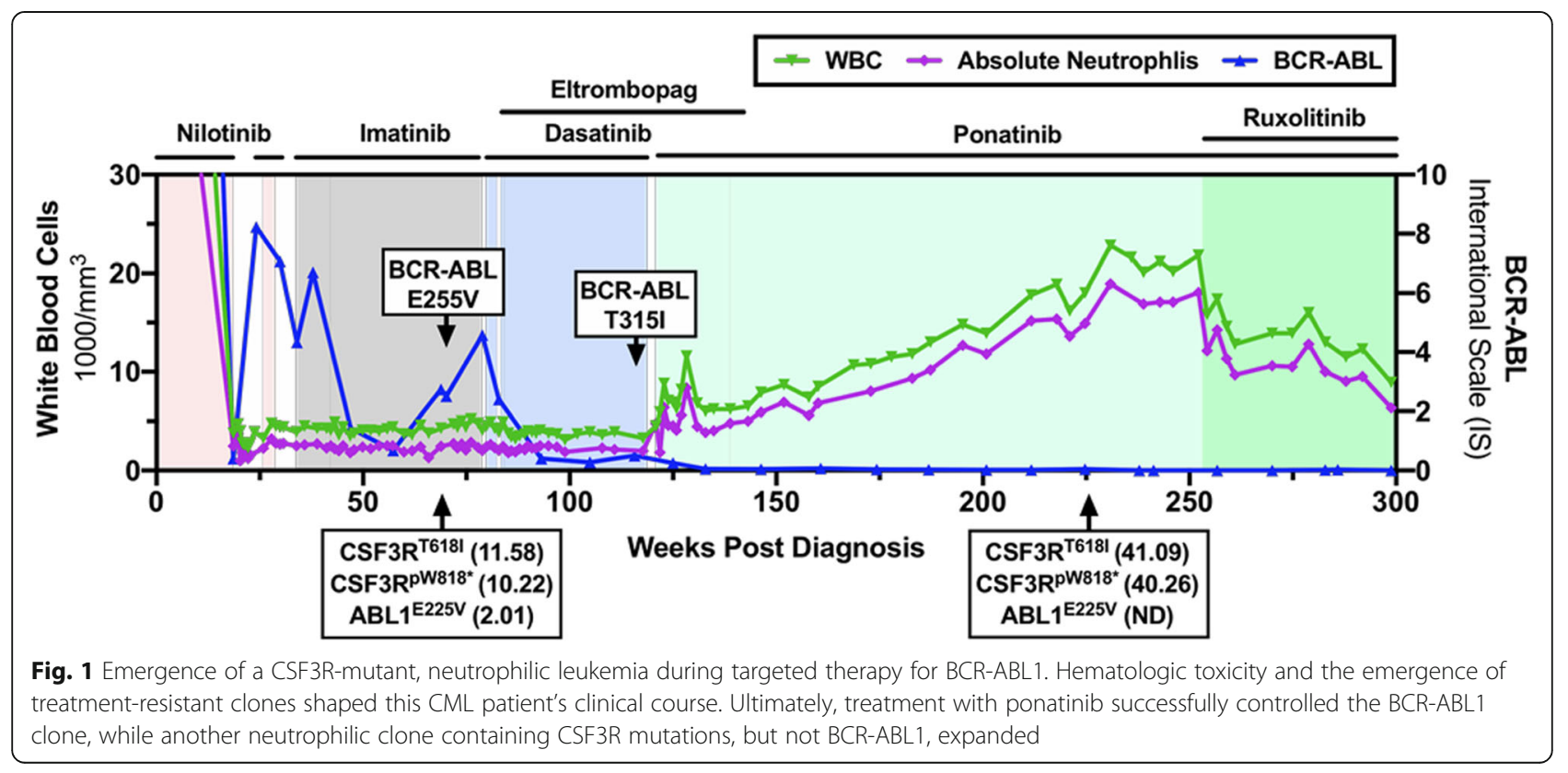


leukemia. It is interesting to note that two granulocytic leukemias arose in this patient, suggesting that either cell intrinsic or microenvironmental factors caused a specific predilection for malignancies of the granulocytic lineage. Germline mutations have not been accessed for this patient. This case describes the exceedingly rare cooccurrence of CML and CNL in the same individual and is an unusual example of clonal evolution under selective pressure from targeted therapy. These findings highlight that myeloid disease with complex clonal architecture is prone to molecular evolution. Patients with such molecularly complex disease may ultimately benefit from combination therapy that targets multiple oncogenic pathways.

\section{Abbreviations}

MPNs: Myeloproliferative neoplasms; CML: Chronic myeloid leukemia; CNL: Chronic neutrophilic leukemia; Ph: Philadelphia; FISH: Chromosome, Fluorescent In Situ Hybridization; IS: International scale; TKIs: Tyrosine kinase inhibitors

\section{Acknowledgements}

Authors thank colleagues at Arizona Center for Cancer Care and Grande Ronde Hospital for collaboration on the care of the patient.

\section{Authors' contributions}

DB and BJD treated the patient; SAC, TPB and JEM analyzed and/or interpreted the data; SAC and TPB wrote the paper. All authors reviewed and approved the manuscript.

\section{Funding}

SAC is supported by a fellowship from the National Cancer Institute, F32CA239422. JEM is supported by an American Society of Hematology Scholar Award, a Gilead Research Scholars Award, and American Cance Society RSG-19-184-01-LIB. TPB is supported by KL2 TR002370-03 and K08 CA245224-01. BJD is supported by Howard Hughes Medical Institute and R01 CA065823-24

\section{Availability of data and materials}

Data sharing requests should be sent to the corresponding author.

\section{Ethics approval and consent to participate}

The OHSU IRB determined that the proposed activity is not research involving human subjects. Access and use of protected health information complies with OHSU HIPAA policies for non-research activities.

\section{Consent for publication}

Not applicable. Data presented in this study has been de-identified and contains none of the 18 HIPPA individual identifiers.

\section{Competing interests}

B.J.D. potential competing interests-SAB: Aileron Therapeutics, ALLCRON, Cepheid, Vivid Biosciences, Celgene, RUNX1 Research Program, EnLiven Therapeutics, Gilead Sciences (inactive), Baxalta (inactive), Monojul (inactive); SAB and stock: Aptose Biosciences, Blueprint Medicines, Beta Cat, Third Coast Therapeutics, GRAlL (inactive), CTI BioPharma (inactive); scientific founder: MolecularMD (inactive, acquired by ICON); board of directors and stock: Amgen; board of directors: Burroughs Wellcome Fund, CureOne; joint steering committee: Beat AML LLS; founder: VP Therapeutics; clinical trial funding: Novartis, Bristol-Myers Squibb, Pfizer; royalties from Patent 6958335 (Novartis exclusive license) and OHSU and Dana- Farber Cancer Institute (one Merck exclusive license). B.J.D. potential competing interests- consultant: Monojul, Patient True Talk; SAB: Aileron Therapeutics, ALLCRON, Cepheid, Gilead Sciences, Vivid Biosciences, Celgene \& Baxalta (inactive); SAB and stock: Aptose Biosciences, Blueprint Medicines, Beta Cat, GRAIL, Third Coast Therapeutics, CTI Bio-Pharma (inactive); scientific founder and stock: MolecularMD; board of directors and stock: Amgen; board of directors: Burroughs
Wellcome Fund, CureOne; joint steering committee: Beat AML LLS; clinical trial funding: Novartis, Bristol-Myers Squibb, Pfizer; royalties: OHSU \#606Novartis exclusive license, OHSU \#2573; Dana-Farber Cancer Institute \#2063Merck exclusive license. J.E.M. receives grant funding from the Gilead Research Scholars Program. The remaining authors declare no competing interests.

Received: 23 October 2020 Accepted: 18 January 2021

Published online: 30 January 2021

\section{References}

1. Sawyers CL. Chronic myeloid leukemia. N Engl J Med. 1999;340(17):1330-40.

2. Lugo TG, Pendergast AM, Muller AJ, Witte ON. Tyrosine kinase activity and transformation potency of bcr-abl oncogene products. Science. 1990; 247(4946):1079-82.

3. Daley GQ, Van Etten RA, Baltimore D. Induction of chronic myelogenous leukemia in mice by the P210bcr/abl gene of the Philadelphia chromosome. Science. 1990;247(4944):824-30.

4. O'Hare T, Walters DK, Stoffregen EP, Jia T, Manley PW, Mestan J, et al. In vitro activity of Bcr-Abl inhibitors AMN107 and BMS-354825 against clinically relevant imatinib-resistant Abl kinase domain mutants. Cancer Res. 2005; 65(11):4500-5.

5. Muller MC, Cortes JE, Kim DW, Druker BJ, Erben P, Pasquini R, et al. Dasatinib treatment of chronic-phase chronic myeloid leukemia: analysis of responses according to preexisting BCR-ABL mutations. Blood. 2009;114(24): 4944-53.

6. Hughes T, Saglio G, Branford S, Soverini S, Kim DW, Muller MC, et al. Impact of baseline BCR-ABL mutations on response to nilotinib in patients with chronic myeloid leukemia in chronic phase. J Clin Oncol. 2009;27(25):420410.

7. Cortes JE, Kantarjian HM, Brummendorf TH, Kim DW, Turkina AG, Shen ZX, et al. Safety and efficacy of bosutinib (SKI-606) in chronic phase Philadelphia chromosome-positive chronic myeloid leukemia patients with resistance or intolerance to imatinib. Blood. 2011;118(17):4567-76.

8. Cortes JE, Kim DW, Pinilla-lbarz J, le Coutre P, Paquette R, Chuah C, et al. A phase 2 trial of ponatinib in Philadelphia chromosome-positive leukemias. N Engl J Med. 2013:369(19):1783-96.

9. Redaelli S, Piazza R, Rostagno R, Magistroni V, Perini P, Marega M, et al. Activity of bosutinib, dasatinib, and nilotinib against 18 imatinib-resistant BCR/ABL mutants. J Clin Oncol. 2009;27(3):469-71.

10. Cortes JE, Kantarjian H, Shah NP, Bixby D, Mauro MJ, Flinn I, et al. Ponatinib in refractory Philadelphia chromosome-positive leukemias. N Engl J Med. 2012;367(22):2075-88.

11. Hochhaus A, Breccia M, Saglio G, Garcia-Gutierrez V, Rea D, Janssen J, et al. Expert opinion-management of chronic myeloid leukemia after resistance to second-generation tyrosine kinase inhibitors. Leukemia. 2020;34:1495502. https://doi.org/10.1038/s41375-020-0842-9.

12. Pardanani A, Lasho TL, Laborde RR, Elliott M, Hanson CA, Knudson RA, et al. CSF3R T6181 is a highly prevalent and specific mutation in chronic neutrophilic leukemia. Leukemia. 2013;27(9):1870-3.

13. Dao KT, Gotlib J, Deininger MMN, Oh ST, Cortes JE, Collins RH Jr, et al. Efficacy of Ruxolitinib in patients with chronic Neutrophilic leukemia and atypical chronic myeloid leukemia. J Clin Oncol. 2020;38(10):1006-18.

14. Maxson JE, Gotlib J, Pollyea DA, Fleischman AG, Agarwal A, Eide CA, et al, Oncogenic CSF3R mutations in chronic neutrophilic leukemia and atypical CML. N Engl J Med. 2013;368(19):1781-90.

\section{Publisher's Note}

Springer Nature remains neutral with regard to jurisdictional claims in published maps and institutional affiliations. 